Review

\title{
American marsupials chromosomes: Why study them?
}

\author{
Marta Svartman \\ Departamento de Biologia Geral, Instituto de Ciências Biológicas, Universidade Federal de Minas Gerais, \\ Belo Horizonte, $M G$, Brazil.
}

\begin{abstract}
Marsupials, one of the three main groups of mammals, are only found in Australia and in the American continent. Studies performed in Australian marsupials have demonstrated the great potential provided by the group for the understanding of basic genetic mechanisms and chromosome evolution in mammals. Genetic studies in American marsupials are relatively scarce and cytogenetic data of most species are restricted to karyotype descriptions, usually without banding patterns. Nevertheless, the first marsupial genome sequenced was that of Monodelphis domestica, a South American species. The knowledge about mammalian genome evolution and function that resulted from studies on $M$. domestica is in sharp contrast with the lack of genetic data on most American marsupial species. Here, we present an overview of the chromosome studies performed in marsupials with emphasis on the South American species.
\end{abstract}

Key words: marsupials, cytogenetics, chromosome evolution.

Received: December 19, 2008; Accepted: June 17, 2009.

\section{Marsupials: Evolutionary History and Taxonomy}

The class Mammalia is traditionally divided into three main groups: Prototheria, comprising only three species of extant monotremes, Metatheria, in which all marsupials are grouped, and Eutheria, that reunites all the other 18 mammalian orders.

Besides typical mammalian features, as homeothermy, hairs and mammary glands, marsupials have biological characteristics that allowed their separation as a distinct group. Among them, which include dental, osteological, cranial and brain features, those related to the reproductive system are the most important. These include a bifid reproductive tract in females, which have double vagina and uterus, and an incomplete placenta. The gestation time is relatively short and the newborns are in an almost embryonic stage. The marsupium or pouch, used to name the group, is not its most important feature and is absent in several species (Stonehouse, 1977; Nowak, 1991).

A recent estimate which included molecular data from almost all extant mammalian groups led BinindaEmonds et al. (2007) to propose that the split of Monotremata from the common mammalian ancestor occurred at 166.2 million years ago (mya), that the lineage of the marsupials would have diverged at 147.7 mya and that the four recognized placental superorders would have originated

Send correspondence to Marta Svartman. Departamento de Biologia Geral, Instituto de Ciências Biológicas, Universidade Federal de Minas Gerais, Av. Antônio Carlos 6627, Pampulha, 31270-910 Belo Horizonte, MG, Brazil. E-mail: svartmanm@icb.ufmg.br. around 100 mya. The diversification of the marsupials was dated at 82.5 mya.

Regarding their evolutionary history, the traditional hypothesis was that marsupials originated during the Cretaceous in North America. From there, they would have migrated to South America, Antarctica and Australia (Clemens, 1977). Marsupial fossils were also found in Europe, Asia and North Africa, where there are no extant representatives of the group, indicating a broader geographical distribution in the past (Nowak, 1991). The discovery of the metatherian fossil Sinodelphys szalayi in northeastern China in a site dated at 125 mya changed this picture (Luo et al., 2003). Eutherian fossils have also been found in the same site, including Eomaia, the earliest known member of the eutherian lineage. Thus the marsupial-placental split must have been no later than 125 mya. Sinodelphys was found to be basal in the metatherian tree, which led Luo et al. (2003) to propose a Eurasian origin for both eutherians and metatherians. From Eurasia, marsupials would have migrated to North America and later to South America and then Antarctica and Australia.

The Australian groups of marsupials are phylogenetically and immunologically more closely related between them than they are to any American group. This led to the suggestion that they originated from a single ancestral stock that would have reached Australia in the beginning of the Tertiary (64 mya), coming from South America via Antarctica (Dawson, 1983). The phylogenetic relationship between the South American monito del monte Dromiciops gliroides and the Australasian marsupials is of special in- 
terest. The traditional view is that Australidelphia, a panGondwanan clade that includes all extant Australian marsupials and the South American microbiotheres, represented presently only by Dromiciops gliroides, would have originated in South America.

Australian marsupials are believed to have reached that continent at the end of the Cretaceous (64 mya) and there they experienced an extensive radiation, which is suggested by the present diversity of the group in Australia. The oldest metatherian fossils known from Australia are from a single site dated at around 55 mya. The recent finding of fairly complete fossils from the metatherian Djarthia murgonenis led Beck et al. (2008) to propose that Djarthia was a member of Australidelphia. A phylogenetic analysis led to the conclusion that Djartia is the most plesiomorphic known australidelphian and may represent the ancestral morphotype of the Australian marsupial radiation. Beck et al. (2008) thus concluded that, contrary to the long held view, the South American microbiotheres may have resulted from back dispersal from marsupials from eastern Gondwana.

Marsupials have been traditionally grouped in the order Marsupialia, but the recognition of their great diversity is reflected in the increasing number of taxa proposed by different authors (Nowak, 1991; Wilson and Reeder, 2005). More recently new classifications were proposed mainly based on molecular data and the most current includes ten orders, seven of which with living representatives (Wilson and Reeder, 2005). These seven orders reunite 331 extant species, from which 237 inhabit Australasia and 94 occur in the American continent (Wilson and Reeder, 2005). The American marsupial fauna is predominantly found in Central and South America, as the only marsupial species in North America, Didelphis virginiana, is a relatively recent invader, derived from the South American D. marsupialis (Dawson, 1983).

Three marsupial orders each including one extant family are found in the American continent. Among them, the family Didelphidae, order Didelphimorphia, is the richest in number of taxa and has the broadest geographical distribution, with representatives in the three Americas. It has 87 species belonging to 17 genera (Caluromys, Caluromysiops, Chironectes, Didelphis, Glironia, Gracilinanus, Hyladelphys, Lestodelphys, Lutreolina, Marmosa, Marmosops, Metachirus, Micoureus, Monodelphis, Philander, Thylamys and Tlacuatzin). Six species, belonging to three genera (Caenolestes, Lestoros e Rhyncolestes), form the family Caenolestidae, order Paucituberculata, whose representatives inhabit high altitude regions, usually in the Andes; the family Microbiotheriidae, order Microbiotheria, has as its sole extant representative Dromiciops gliroides, which has only been found in southern Chile and in the adjacent region of Argentina (Wilson and Reeder, 2005).

\section{The Chromosomes of American Marsupials}

Marsupials were one of the first mammalian groups to have their chromosomes studied. In his review, Hayman (1990) listed the diploid numbers of 178 species, which varies from $2 \mathrm{n}=10$ in Pseudocheirus cupreus to $2 \mathrm{n}=32$ in Aepyprymnus rufescens, both from Australia.

From the 94 currently recognized American marsupial species (Wilson and Reeder, 2005), 45 had their karyotypes reported, most of them without banding patterns (Table 1). Three diploid numbers have been found in American marsupials: $2 \mathrm{n}=22$ in species of Didelphis, Chironectes, Philander and Lutreolina, $2 \mathrm{n}=18$ in species of Monodelphis, and $2 \mathrm{n}=14$ in all other species studied, which belong to the genera Caluromys, Gracilinanus, Marmosa, Metachirus, Thylamys, Micoureus, Marmosops, Tlacuatzin, Dromiciops, Caenolestes, Lestoros and Rhyncolestes.

The current available chromosome data for American marsupials are detailed in Table 1. In this Table, the fundamental number (FN) seems to be variable both within the same species, for instance Caluromys lanatus, and among species with the same diploid number, as the Monodelphis species. A closer examination of the literature reveals that there is no real variation in autosomal arms in each of the species studied by each author. The reason for the difference is that some authors counted the short arms of the acrocentric or subtelocentric chromosomes, while others did not. When these short arms are not included, the FN count is very constant and equal to 20 in all species with $2 \mathrm{n}$ $=14$, except for all the Marmosops species, the caenolestids and the microbiotherid, which exhibit a FN $=24$. The difference in the FN between these two karyotypic forms is due to the two smaller autosome pairs, which are acrocentric in the $\mathrm{FN}=20$ and metacentric/submetacentric in the $\mathrm{FN}=24$ complements. The same explanation applies to the $2 \mathrm{n}=18$ Monodelphis: all the species studied presented two clearly biarmed elements, resulting in a $\mathrm{FN}=20$.

This observation means that no real variation was observed between the conventionally stained autosomes of specimens of the same species collected in different localities and studied by various authors. Moreover, no variation in the autosomes seems detectable among species with each of the three diploid numbers $(2 \mathrm{n}=14,18$ and 22$)$, except for the species with $2 n=14$ and $F N=24$.

Great intraspecific conservation was also observed in the sex chromosomes. Many apparent differences are due to the nomenclature used by various authors ( $\mathrm{SM}$ or $\mathrm{M}$; $\mathrm{T}$ or A) or by technical difficulties because of the low quality of the chromosome preparations, especially in the older reports. The sex chromosomes are always the smallest ones in the karyotypes of the American marsupials studied and some species have very small $\mathrm{Y}$ chromosomes, considered as dot-like by some or as very small A by others. Due to their small size, determining their morphology may be dif- 
Table 1 - American marsupial species karyotyped.

\begin{tabular}{|c|c|c|c|c|c|c|c|c|}
\hline Species $^{\mathrm{a}}$ & Referred as & $2 n$ & FN & $\mathrm{X}$ & $\mathrm{Y}$ & Banding and FISH & Provenience $^{b}$ & Reference \\
\hline \multicolumn{9}{|c|}{ Family Didelphidae, Order Didelphimorphia } \\
\hline \multirow[t]{2}{*}{ Caluromys derbianus } & & 14 & 24 & A & $\mathrm{T}$ & & Nicaragua & Biggers et al., 1965 \\
\hline & & 14 & 24 & A & A & & $\begin{array}{l}\text { Cartago, Costa Rica; Chia- } \\
\text { pas, Mexico }\end{array}$ & Reig et al., 1977 \\
\hline \multirow{7}{*}{\multicolumn{2}{|c|}{ Caluromys lanatus }} & 14 & 24 & SM & - & & Iquitos, Peru & Hayman and Martin, 1974 \\
\hline & & 14 & 24 & A & $\mathrm{A}$ & & Loreto, Peru & Reig et al., 1977 \\
\hline & & 14 & 22 & M & - & $\mathrm{G}, \mathrm{C}, \mathrm{Ag}-\mathrm{NORs}$ & San Diego, Zoo, USA & Rofe and Hayman, 1985 \\
\hline & & 14 & 22 & SM & $\mathrm{A}$ & G, Ag-NORs & Amazonas, Brazil & Casartelli et al., 1986 \\
\hline & & 14 & 20 & A & $\mathrm{D}$ & $\mathrm{G}, \mathrm{Ag}$-NORs & Rondônia, Brazil & Souza et al., 1990 \\
\hline & & 14 & 22 & $\mathrm{~A}$ & M & & Pando, Bolivia & Palma and Yates, 1996 \\
\hline & & 14 & 20 & SM & $\mathrm{D}$ & Ag-NORs & Goiás, Brazil & Pereira et al., 2008 \\
\hline \multirow{5}{*}{\multicolumn{2}{|c|}{ Caluromys philander }} & 14 & 24 & A & - & & not specified & Hayman and Martin, 1974 \\
\hline & & 14 & 24 & SM & A & & $\begin{array}{l}\text { Simla, Trindad; Guárico, } \\
\text { Aragua and Miranda, Vene- } \\
\text { zuela }\end{array}$ & Reig et al., 1977 \\
\hline & & 14 & 20 & A & $\mathrm{D}$ & G, C, Ag-NORs & Pernambuco, Brazil & Souza et al., 1990 \\
\hline & & 14 & 20 & A & A & $\begin{array}{l}\text { G, Ag-NORs, FISH } \\
\text { (Tel; rDNA) }\end{array}$ & São Paulo, Brazil & $\begin{array}{l}\text { Svartman and Vianna-Mor- } \\
\text { gante, 1998, 1999, } 2003\end{array}$ \\
\hline & & 14 & 20 & A & $\mathrm{D}$ & Ag-NORs & São Paulo, Brazil & Pereira et al., 2008 \\
\hline \multirow{2}{*}{\multicolumn{2}{|c|}{ Gracilinanus agilis }} & 14 & 24 & SM & SM & & La Paz, Bolivia & Palma and Yates, 1996 \\
\hline & & 14 & 24 & M & A & & $\begin{array}{l}\text { Goiás and Minas } \\
\text { Gerais, Brazil }\end{array}$ & Carvalho et al., 2002 \\
\hline \multirow{2}{*}{\multicolumn{2}{|c|}{ Gracilinanus emiliae }} & 14 & 24 & $\mathrm{M} / \mathrm{A}^{*}$ & A & $\begin{array}{l}\text { C, Ag-NORs, FISH } \\
\text { (Tel) }\end{array}$ & Goiás, Brazil & $\begin{array}{l}\text { Carvalho and Mattevi, 2000; } \\
\text { Carvalho et al., } 2002\end{array}$ \\
\hline & & 14 & 24 & SM & A & $\mathrm{Ag}$-NORs & Goiás, Brazil & Pereira et al., 2008 \\
\hline \multirow{3}{*}{\multicolumn{2}{|c|}{$\begin{array}{l}\text { Gracilinanus } \\
\text { microtarsus }\end{array}$}} & 14 & 24 & SM & A & $\begin{array}{l}\text { C, Ag-NORs, FISH } \\
\text { (Tel) }\end{array}$ & Rio Grande do Sul, Brazil & $\begin{array}{l}\text { Carvalho and Mattevi, 2000; } \\
\text { Carvalho et al., } 2002\end{array}$ \\
\hline & & 14 & 20 & A & A & & Bahia, Brazil & Pereira and Geise, 2007 \\
\hline & & 14 & 24 & $\mathrm{M}$ & - & & São Paulo, Brazil & Pereira et al., 2008 \\
\hline Marmosa mexicana & & 14 & 24 & A & $\mathrm{D}$ & & Nicaragua & Biggers et al., 1965 \\
\hline \multirow[t]{8}{*}{ Marmosa murina } & & 14 & 24 & M & A & & Villavicencio, Colombia & Hayman and Martin, 1974 \\
\hline & & 14 & 24 & M & $\mathrm{A}$ & & $\begin{array}{l}\text { Loreto, Peru; Bolivar, } \\
\text { Venezuela }\end{array}$ & Reig et al., 1977 \\
\hline & & 14 & 20 & A & $\mathrm{D}$ & G, C, Ag-NORs & Pernambuco, Brazil & Souza et al., 1990 \\
\hline & & 14 & 24 & $\mathrm{M} / \mathrm{A}^{*}$ & A & $\begin{array}{l}\text { C, Ag-NORs, FISH } \\
\text { (Tel) }\end{array}$ & $\begin{array}{l}\text { Amapá, Goiás and } \\
\text { Tocantins, Brazil }\end{array}$ & $\begin{array}{l}\text { Carvalho and Mattevi, 2000; } \\
\text { Carvalho et al., } 2002\end{array}$ \\
\hline & & 14 & 20 & A & - & C, FISH (Tel) & $\begin{array}{l}\text { Ceará and Mato } \\
\text { Grosso, Brazil }\end{array}$ & Pagnozzi et al., 2002 \\
\hline & & 14 & 22 & A & - & Ag-NORs & Tocantins, Brazil & Lima, 2004 \\
\hline & & 14 & 20 & A & $\mathrm{D}$ & & Espírito Santo, Brazil & Paresque et al., 2004 \\
\hline & & 14 & 20 & SM & A & Ag-NORs & Goiás and Tocantins, Brazil & Pereira et al., 2008 \\
\hline \multirow{2}{*}{\multicolumn{2}{|c|}{ Marmosa robinsoni }} & 14 & 20 & M & $\mathrm{T}$ & & $\begin{array}{l}\text { Guárico and Apure, Vene- } \\
\text { zuela }\end{array}$ & Reig, 1968 \\
\hline & & 14 & 24 & SM & A & & $\begin{array}{l}\text { Guárico, Apure and } \\
\text { Miranda, Venezuela }\end{array}$ & Reig et al., 1977 \\
\hline \multicolumn{2}{|l|}{ Marmosops dorothea } & 14 & 24 & $\mathrm{M}$ & A & & La Paz, Bolivia & Palma and Yates, 1996 \\
\hline \multirow[t]{2}{*}{ Marmosops fuscatus } & Marmosa fuscata & 14 & 24 & M & A & & Northern Venezuela & Reig and Sonneschein, 1970 \\
\hline & Marmosa fuscata & 14 & 24 & M & A & & $\begin{array}{l}\text { Aragua and Miranda, Vene- } \\
\text { zuela }\end{array}$ & Reig et al., 1977 \\
\hline \multirow{5}{*}{\multicolumn{2}{|c|}{ Marmosops incanus }} & 14 & 24 & M & A & $\begin{array}{l}\text { G, C, Ag-NORs, FISH } \\
\text { (Tel; rDNA; Gen) }\end{array}$ & São Paulo, Brazil & $\begin{array}{l}\text { Svartman and Vianna-Mor- } \\
\text { gante, 1998, 1999, } 2003\end{array}$ \\
\hline & & 14 & 24 & M & A & C, Ag-NORs & $\begin{array}{l}\text { Bahia and Minas } \\
\text { Gerais, Brazil }\end{array}$ & Carvalho et al., 2002 \\
\hline & & 14 & 24 & M & - & C, FISH (Tel) & Bahia and São Paulo, Brazil & Pagnozzi et al., 2002 \\
\hline & & 14 & 24 & M & A & & Espírito Santo, Brazil & Paresque et al., 2004 \\
\hline & & 14 & 24 & M & A & & Bahia, Brazil & Pereira and Geise, 2007 \\
\hline \multicolumn{2}{|l|}{ Marmosops noctivagus } & 14 & 24 & SM & UN & & La Paz, Bolivia & Palma and Yates, 1996 \\
\hline \multirow[t]{3}{*}{ Marmosops parvidens } & & 14 & 24 & SM & - & & La Paz, Bolivia & Palma and Yates, 1996 \\
\hline & & 14 & 24 & SM & A & Ag-NORs & Goiás, Brazil & Carvalho et al., 2002 \\
\hline & & 14 & 24 & M & A & C, FISH (Tel) & Mato Grosso, Brazil & Pagnozzi et al., 2002 \\
\hline
\end{tabular}


Table 1 (cont.)

\begin{tabular}{|c|c|c|c|c|c|c|c|c|}
\hline Species $^{\mathrm{a}}$ & Referred as & $2 n$ & FN & $\mathrm{X}$ & $\mathrm{Y}$ & Banding and FISH & Provenience $^{\mathrm{b}}$ & Reference \\
\hline \multicolumn{2}{|c|}{ Marmosops paulensis } & 14 & 24 & M & A & Ag-NORs & São Paulo, Brazil & Pereira et al., 2008 \\
\hline \multirow{8}{*}{\multicolumn{2}{|c|}{ Metachirus nudicaudatus }} & 14 & 20 & A & - & & Kasmera, Venezuela & Hayman and Martin, 1974 \\
\hline & & 14 & 20 & A & A & $\mathrm{C}$ & Meta, Colombia & Yunis et al., 1973 \\
\hline & & 14 & 24 & A & A & & $\begin{array}{l}\text { Loreto and Ayacucho, } \\
\text { Peru;Trujillo and Mé- } \\
\text { rida, Venezuela }\end{array}$ & Reig et al., 1977 \\
\hline & & 14 & 20 & A & - & & La Paz, Bolivia & Palma and Yates, 1996 \\
\hline & & 14 & 20 & A & A & $\begin{array}{l}\text { G, C, Ag-NORs, FISH } \\
\text { (Tel; rDNA) }\end{array}$ & São Paulo, Brazil & $\begin{array}{l}\text { Svartman and Vianna-Mor- } \\
\text { gante, 1998, 1999, } 2003\end{array}$ \\
\hline & & 14 & 20 & A & A & C, FISH (Tel) & Mato Grosso, Brazil & Pagnozzi et al., 2002 \\
\hline & & 14 & 20 & $\mathrm{~A}$ & $\mathrm{~A}$ & & Espírito Santo, Brazil & Paresque et al., 2004 \\
\hline & & 14 & 20 & A & - & Ag-NORs & São Paulo, Brazil & Pereira et al., 2008 \\
\hline \multirow[t]{2}{*}{ Micoureus alstoni } & Marmosa alstoni & 14 & & & & & & Hsu and Bernischke, $1971^{c}$ \\
\hline & Marmosa alstoni & 14 & 24 & A & A & & San Jose, Costa Rica & Reig et al., 1977 \\
\hline \multicolumn{2}{|c|}{ Micoureus constantiae } & 14 & 20 & A & A & & Pando, Bolivia & Palma and Yates, 1996 \\
\hline \multicolumn{2}{|c|}{ Micoureus demerarae } & 14 & 20 & $\mathrm{~A}$ & - & & Rancho Grande, Venezuela & Hayman and Martin, 1974 \\
\hline & Marmosa cinerea & 14 & 24 & M & A & & $\begin{array}{l}\text { Loreto, Peru; Aragua, Boli- } \\
\text { var, Amazonas and Mérida, } \\
\text { Venezuela }\end{array}$ & Reig et al., 1977 \\
\hline & Marmosa cinerea & 14 & 20 & A & A & G, C, Ag-NORs & Amazonas, Brazil & Casartelli et al., 1986 \\
\hline & Marmosa cinerea & 14 & 20 & $\mathrm{~A}$ & $\mathrm{~A}$ & $\mathrm{G}, \mathrm{C}, \mathrm{Ag}-\mathrm{NORs}$ & Pernambuco, Brazil & Souza et al., 1990 \\
\hline & \multirow[t]{6}{*}{ Micoureus cinereus } & 14 & 20 & $\mathrm{~A}$ & - & & La Paz, Bolivia & Palma and Yates, 1996 \\
\hline & & 14 & 20 & A & A & $\begin{array}{l}\text { G, C, Ag-NORs, FISH } \\
\text { (Tel; rDNA, Gen) }\end{array}$ & São Paulo, Brazil & $\begin{array}{l}\text { Svartman and Vianna-Mor- } \\
\text { gante, 1998, 1999, } 2003\end{array}$ \\
\hline & & 14 & 20 & $\mathrm{~A}$ & A & C, FISH (Tel) & $\begin{array}{l}\text { Bahia, Ceará, Goiás, Mato } \\
\text { Grosso and São Paulo, Brazil }\end{array}$ & Pagnozzi et al., 2000 \\
\hline & & 24 & 24 & A & A & $\begin{array}{l}\text { C, Ag-NORs, FISH } \\
\text { (Tel) }\end{array}$ & $\begin{array}{l}\text { Goiás and Rio Grande do } \\
\text { Sul, Brazil }\end{array}$ & $\begin{array}{l}\text { Carvalho and Mattevi, } 2000 \\
\text { Carvalho et al., } 2002\end{array}$ \\
\hline & & 14 & 20 & A & A & & Espírito Santo, Brazil & Paresque et al., 2004 \\
\hline & & 14 & 20 & $\mathrm{~A}$ & - & Ag-NORs & São Paulo, Brazil & Pereira et al., 2008 \\
\hline \multicolumn{2}{|c|}{ Micoureus paraguayanus } & 14 & 20 & $\mathrm{~A}$ & - & Ag-NORs & São Paulo, Brazil & Pereira et al., 2008 \\
\hline \multirow[t]{5}{*}{ Thylamys elegans } & Marmosa elegans & 14 & 24 & SM & $\mathrm{D}$ & & Aconcagua, Chile & Reig et al., 1972 \\
\hline & Marmosa elegans & 14 & 22 & SM & $\mathrm{A}$ & & $\begin{array}{l}\text { Aconcagua, Chile; Huanca- } \\
\text { velica and Arequipa, Peru }\end{array}$ & Reig et al., 1977 \\
\hline & Marmosa elegans & 14 & 24 & A & - & Ag-NORs & Coquimbo, Chile & $\begin{array}{l}\text { Fernandez-Donoso et al., } \\
1979\end{array}$ \\
\hline & & 14 & 20 & A & UN & & $\begin{array}{l}\text { Cochabamba and Santa } \\
\text { Cruz, Bolivia }\end{array}$ & Palma and Yates, 1996 \\
\hline & & 14 & 20 & ST & - & $\mathrm{G}, \mathrm{C}$ & $\begin{array}{l}\text { Rio Loa, Pichidangui and } \\
\text { Las Melosas, Chile }\end{array}$ & Spotorno et al., 1997 \\
\hline Thylamys macrurus & T. macrura & 14 & 20 & A & - & & Concepción, Paraguay & Palma, 1995 \\
\hline \multirow[t]{2}{*}{ Thylamys pallidior } & & 14 & 20 & $\mathrm{~A}$ & $\mathrm{UN}$ & & $\begin{array}{l}\text { Chuquisaca and Tarija, } \\
\text { Bolivia }\end{array}$ & Palma, 1995 \\
\hline & & 14 & 20 & $\mathrm{~A}$ & $\mathrm{UN}$ & & Chuquisaca, Bolivia & Palma and Yates, 1996 \\
\hline \multirow[t]{2}{*}{ Thylamys pusillus } & Marmosa pusilla & 14 & 24 & M & $\mathrm{D}$ & & Buenos Aires, Argentina & Reig et al., 1977 \\
\hline & & 14 & 20 & SM & - & & Tarija, Bolivia & Palma and Yates, 1996 \\
\hline \multicolumn{2}{|l|}{ Thylamys velutinus } & 14 & 24 & SM & A & & Goiás, Brazil & Carvalho et al., 2002 \\
\hline \multirow{4}{*}{\multicolumn{2}{|c|}{ Monodelphis americana }} & 18 & 22 & $\mathrm{~T}$ & - & & Paraíba, Brazil & Langguth and Lima, 1988 \\
\hline & & 18 & 22 & A & A & C, FISH (Tel) & Ceará, Brazil & Pagnozzi et al., 2002 \\
\hline & & 18 & 32 & A & A & & Espírito Santo, Brazil & Paresque et al., 2004 \\
\hline & & 18 & 32 & $\mathrm{~A}$ & - & & São Paulo, Brazil & Pereira et al., 2008 \\
\hline \multirow[t]{5}{*}{$\begin{array}{l}\text { Monodelphis } \\
\text { brevicaudata }\end{array}$} & & 18 & 20 & A & $\mathrm{D}$ & & $\begin{array}{l}\text { Aragua and Zulia, Vene- } \\
\text { zuela }\end{array}$ & Reig and Bianchi, 1969 \\
\hline & & 18 & 30 & A & A & & $\begin{array}{l}\text { Aragua, Merida and Zu- } \\
\text { lia, Venezuela }\end{array}$ & Reig et al., 1977 \\
\hline & M. orinoci & 18 & 30 & A & A & & Guárico, Venezuela & Reig et al., 1977 \\
\hline & & 18 & 22 & A & $\mathrm{D}$ & & Pando, Bolivia & Palma and Yates, 1996 \\
\hline & & 18 & 30 & A & $\mathrm{D}$ & $\begin{array}{l}\text { C, Ag-NORs, FISH } \\
\text { (Tel) }\end{array}$ & Amapá and Roraima, Brazil & $\begin{array}{l}\text { Carvalho and Mattevi, } 2000 \\
\text { Carvalho et al., } 2002\end{array}$ \\
\hline
\end{tabular}


Table 1 (cont.)

\begin{tabular}{|c|c|c|c|c|c|c|c|}
\hline Referred as & $2 n$ & FN & $X$ & Y & Banding and FISH & Provenience $^{\mathrm{b}}$ & Reference \\
\hline \multirow[t]{3}{*}{ Monodelphis dimidiata } & 18 & 20 & A & D & & Buenos Aires, Argentina & Reig and Bianchi, 1969 \\
\hline & 18 & 30 & $\mathrm{~A}$ & A & & Buenos Aires, Argentina & Reig et al., 1977 \\
\hline & 18 & 32 & SM & $\mathrm{D}$ & C, Ag-NORs & Rio Grande do Sul, Brazil & Carvalho et al., 2002 \\
\hline \multirow[t]{9}{*}{ Monodelphis domestica } & 18 & 20 & A & A & G, C, NOR & & Merry et al., 1983 \\
\hline & 18 & 20 & A & A & $\mathrm{G}$ & Laboratory bred, USA & Pathak et al., 1993 \\
\hline & 18 & 24 & A & A & & $\begin{array}{l}\text { Chuquisaca and Santa } \\
\text { Cruz, Bolivia }\end{array}$ & Palma and Yates, 1996 \\
\hline & 18 & 20 & A & - & $\begin{array}{l}\text { G, C, Ag-NORs, FISH } \\
\text { (Tel; rDNA) }\end{array}$ & Laboratory bred, Brazil & $\begin{array}{l}\text { Svartman and Vianna-Mor- } \\
\text { gante, 1998, 1999, } 2003\end{array}$ \\
\hline & 18 & 28 & A & $\mathrm{D}$ & $\begin{array}{l}\text { C, Ag-NORs, FISH } \\
\text { (Tel) }\end{array}$ & Goiás, Brazil & $\begin{array}{l}\text { Carvalho and Mattevi, } 2000 \\
\text { Carvalho et al., } 2002\end{array}$ \\
\hline & 18 & 20 & $\mathrm{~A}$ & A & C, FISH (Tel) & Ceará and Goiás, Brazil & Pagnozzi et al., 2002 \\
\hline & 18 & 30 & A & $\mathrm{D}$ & & Espírito Santo, Brazil & Paresque et al., 2004 \\
\hline & 18 & 22 & $\mathrm{~A}$ & A & & Bahia, Brazil & Pereira and Geise, 2007 \\
\hline & 18 & 20 & $\mathrm{~A}$ & $\mathrm{~A}$ & & Goiás and Tocantins, Brazil & Pereira et al., 2008 \\
\hline \multirow[t]{2}{*}{ Monodelphis kunsi } & 18 & 30 & SM & SM & & Tarija, Bolivia & Palma and Yates, 1996 \\
\hline & 18 & 30 & SM & A & $\begin{array}{l}\text { C, Ag-NORs, FISH } \\
\text { (Tel) }\end{array}$ & Goiás, Brazil & $\begin{array}{l}\text { Carvalho and Mattevi, } 2000 \\
\text { Carvalho et al., } 2002\end{array}$ \\
\hline Monodelphis rubida & 18 & 32 & $\mathrm{~A}$ & $\mathrm{~A}$ & Ag-NORs & São Paulo, Brazil & Pereira et al., 2008 \\
\hline \multirow[t]{4}{*}{ Chironectes minimus } & 22 & 20 & A & A & & Rio Camoto, Venezuela & Hayman and Martin, 1974 \\
\hline & 22 & 20 & A & A & & not specified & Reig et al., 1977 \\
\hline & 22 & 20 & A & $\mathrm{UN}$ & & La Paz, Bolivia & Palma and Yates, 1996 \\
\hline & 22 & 20 & A & - & $\begin{array}{l}\text { C, Ag-NORs, FISH } \\
\text { (Tel) }\end{array}$ & Goiás, Brazil & $\begin{array}{l}\text { Carvalho and Mattevi, } 2000 \\
\text { Carvalho et al., } 2002\end{array}$ \\
\hline \multirow[t]{7}{*}{ Didelphis albiventris } & 22 & & & & & & Saez, $1931^{\mathrm{c}}$ \\
\hline & 22 & 20 & $\mathrm{~A}$ & $\mathrm{~A}$ & & $\begin{array}{l}\text { Buenos Aires, Argentina; } \\
\text { Mérida, Venezuela; Huana- } \\
\text { co, Peru }\end{array}$ & Reig et al., 1977 \\
\hline & 22 & 20 & A & A & $\mathrm{G}, \mathrm{C}$ & São Paulo, Brazil & $\begin{array}{l}\text { Yonenaga-Yassuda et al., } \\
1982\end{array}$ \\
\hline & 22 & 20 & A & A & $\mathrm{G}, \mathrm{C}, \mathrm{Ag}-\mathrm{NORs}$ & Uruguay (six localities) & Seluja et. al., 1984 \\
\hline & 22 & 20 & A & A & G, C, Ag-NORs & São Paulo, Brazil & Casartelli et al., 1986 \\
\hline & 22 & 20 & $\mathrm{~A}$ & A & & Tarija, Bolivia & Palma and Yates, 1996 \\
\hline & 22 & 20 & A & A & $\begin{array}{l}\text { C, Ag-NORs, FISH } \\
\text { (Tel) }\end{array}$ & Goiás and Tocantins, Brazil & $\begin{array}{l}\text { Carvalho and Mattevi, } 2000 \\
\text { Carvalho et al., } 2002\end{array}$ \\
\hline \multirow[t]{2}{*}{ Didelphis aurita } & 22 & 20 & A & A & & Bahia, Brazil & Carvalho et al., 2002 \\
\hline & 22 & 20 & $\mathrm{~A}$ & $\mathrm{~A}$ & & Espírito Santo, Brazil & Paresque et al., 2004 \\
\hline \multirow[t]{11}{*}{ Didelphis marsupialis } & 22 & & & & & & Dreyfus and Campos, 1941 \\
\hline & 22 & 20 & SM & $\mathrm{T}$ & & Philadelphia, USA & Biggers et al., 1965 \\
\hline & 22 & 20 & $\mathrm{~A}$ & A & & $\begin{array}{l}\text { San Jose, Costa Rica; San } \\
\text { Luis Potosi, Veracruz and } \\
\text { Chiapas, Mexico; Loreto, } \\
\text { Peru; Aragua, Miranda and } \\
\text { Merida, Venezuela }\end{array}$ & Reig et al., 1977 \\
\hline & 22 & 20 & A & A & G, C, Ag-NORs & São Paulo, Brazil & $\begin{array}{l}\text { Yonenaga-Yassuda et al., } \\
1982\end{array}$ \\
\hline & 22 & 20 & A & $\mathrm{A}$ & $\mathrm{G}, \mathrm{Ag}$-NORs & $\begin{array}{l}\text { Amazonas and São } \\
\text { Paulo, Brazil }\end{array}$ & Casartelli et al., 1986 \\
\hline & 22 & 20 & A & A & & La Paz, Bolivia & Palma and Yates, 1996 \\
\hline & 22 & 20 & A & A & $\begin{array}{l}\text { G, C, Ag-NORs, FISH } \\
\text { (Tel; rDNA) }\end{array}$ & São Paulo, Brazil & $\begin{array}{l}\text { Svartman and Vianna-Mor- } \\
\text { gante, 1998, 1999, } 2003\end{array}$ \\
\hline & 22 & 20 & $\mathrm{~A}$ & $\mathrm{~A}$ & $\mathrm{C}, \mathrm{Ag}-\mathrm{NORs}$ & Amapá and Pará & Carvalho et al., 2002 \\
\hline & 22 & 20 & $\mathrm{~A}$ & A & C, FISH (Tel) & Mato Grosso, Brazil & Pagnozzi et al., 2002 \\
\hline & 22 & 20 & A & A & Ag-NORs & Tocantins, Brazil & Lima, 2004 \\
\hline & 22 & 20 & A & - & & Tocantins, Brazil & Pereira et al., 2008 \\
\hline \multirow[t]{3}{*}{ Didelphis virginiana } & 22 & & & & & & Painter, $1922^{\mathrm{c}}$ \\
\hline & 22 & 32 & M & A & $\mathrm{C}$ & Texas, USA & Sinha et al., 1972 \\
\hline & 22 & 32 & M & A & $\begin{array}{l}\mathrm{G}, \mathrm{C}, \mathrm{H}^{3} \text {-timidine } \\
\text { replication }\end{array}$ & not specified & Sinha and Kakati, 1976 \\
\hline
\end{tabular}


Table 1 (cont.)

\begin{tabular}{|c|c|c|c|c|c|c|c|c|}
\hline Species $^{\mathrm{a}}$ & Referred as & $2 n$ & FN & $\mathrm{X}$ & $\mathrm{Y}$ & Banding and FISH & Provenience $^{b}$ & Reference \\
\hline & & 22 & 32 & M & A & & $\begin{array}{l}\text { San Luis Potosi, Nayarit, } \\
\text { Chiapas and Yucatán, } \\
\text { Mexico; Louisiana and Te- } \\
\text { xas, USA }\end{array}$ & Reig et al., 1977 \\
\hline \multirow{7}{*}{\multicolumn{2}{|c|}{ Lutreolina crassicaudata }} & 22 & & & & & & Saez, $1938^{\mathrm{c}}$ \\
\hline & & 22 & 20 & $\mathrm{M}$ & A & & Buenos Aires, Argentina & Reig et al., 1977 \\
\hline & & 22 & 20 & M & A & $\mathrm{G}, \mathrm{C}$ & São Paulo, Brazil & $\begin{array}{l}\text { Yonenaga-Yassuda et al., } \\
1982\end{array}$ \\
\hline & & 22 & 20 & M & A & G, C, Ag-NORs & Uruguay (six localities) & Seluja et. al., 1984 \\
\hline & & 22 & 20 & M & A & & not spec, Bolivia & Palma and Yates, 1996 \\
\hline & & 22 & 20 & M & A & $\begin{array}{l}\text { C, Ag-NORs, FISH } \\
\text { (Tel) }\end{array}$ & Rio Grande do Sul, Brazil & $\begin{array}{l}\text { Carvalho and Mattevi, 2000; } \\
\text { Carvalho et al., } 2002\end{array}$ \\
\hline & & 22 & 20 & $\mathrm{M}$ & - & C, FISH (Tel) & Goiás, Brazil & Pagnozzi et al., 2002 \\
\hline \multirow[t]{3}{*}{ Philander frenatus } & P. frenata & 22 & 20 & A & $\mathrm{A} / \mathrm{M}$ & $\begin{array}{l}\text { C, Ag-NORs, FISH } \\
\text { (Tel) }\end{array}$ & $\begin{array}{l}\text { Santa Catarina and Rio } \\
\text { Grande do Sul, Brazil }\end{array}$ & $\begin{array}{l}\text { Carvalho and Mattevi, 2000; } \\
\text { Carvalho et al., } 2002\end{array}$ \\
\hline & P. frenata & 22 & 20 & A & A & & Espírito Santo, Brazil & Paresque et al., 2004 \\
\hline & P. frenata & 22 & 20 & $\mathrm{~A}$ & $\mathrm{~A}$ & Ag-NORs & São Paulo, Brazil & Pereira et al., 2008 \\
\hline Philander mcilhennyi & & 22 & 20 & A & $\mathrm{A}$ & & Loreto, Peru & Reig et al., 1977 \\
\hline \multirow[t]{7}{*}{ Philander opossum } & & 22 & 20 & $\mathrm{~T}$ & $\mathrm{~T}$ & & Nicaragua & Biggers et al., 1965 \\
\hline & & 22 & 20 & $\mathrm{~A}$ & $\mathrm{~A}$ & & $\begin{array}{l}\text { Cartago, Costa Rica; Chia- } \\
\text { pas, Mexico; Loreto and } \\
\text { Ayacucho, Peru; Barinas, } \\
\text { Venezuela }\end{array}$ & Reig et al., 1977 \\
\hline & & 22 & 20 & A & A & G, C, Ag-NORs & $\begin{array}{l}\text { São Paulo and Rio de Ja- } \\
\text { neiro, Brazil }\end{array}$ & $\begin{array}{l}\text { Yonenaga-Yassuda } \\
\text { et al., } 1982\end{array}$ \\
\hline & & 22 & 20 & A & A & & $\begin{array}{l}\text { Beni, Chuquisaca and Santa } \\
\text { Cruz, Bolivia }\end{array}$ & Palma and Yates, 1996 \\
\hline & & 22 & 20 & $\mathrm{~A}$ & - & $\begin{array}{l}\text { G, C, Ag-NORs, FISH } \\
\text { (Tel; rDNA; Gen) }\end{array}$ & $\begin{array}{l}\text { Espírito Santo and São Pau- } \\
\text { lo, Brazil }\end{array}$ & $\begin{array}{l}\text { Svartman and Vianna-Mor- } \\
\text { gante, } 1998,1999,2003\end{array}$ \\
\hline & & 22 & 20 & A & $\mathrm{A}$ & $\begin{array}{l}\text { C, Ag-NORs, FISH } \\
(\mathrm{Tel})\end{array}$ & Goiás, Brazil & $\begin{array}{l}\text { Carvalho and Mattevi, 2000; } \\
\text { Carvalho et al., } 2002\end{array}$ \\
\hline & & 22 & 20 & A & A & Ag-NORs & Goiás, Brazil & Pereira et al., 2008 \\
\hline Tlacuatzin canescens & Marmosa canescens & 22 & 20 & $\mathrm{~A}$ & $\mathrm{D}$ & & Mexico (four localities) & Engstrom and Gardner, 1988 \\
\hline \multicolumn{9}{|c|}{ Family Caenolestidae, Order Paucituberculata } \\
\hline \multicolumn{2}{|c|}{ Caenolestes fuliginosus } & 14 & 24 & A & $\mathrm{D}$ & & not specified & Hayman et al., $1971^{\mathrm{d}}$ \\
\hline & C. obscurus & 14 & 24 & $\mathrm{~A}$ & $\mathrm{D}$ & & Cauca, Colombia & Hayman et al., 1971 \\
\hline Lestoros inca & & 14 & 24 & A & $\mathrm{D}$ & & Cuzco, Peru & Hayman et al., 1971 \\
\hline Rhyncholestes raphana & arus & 14 & 24 & A & A & & Osorno, Chile & $\begin{array}{l}\text { Gallardo and Patterson, } \\
1987\end{array}$ \\
\hline \multicolumn{9}{|c|}{ Family Microbiotheriidae, Order Microbiotheria } \\
\hline \multirow[t]{5}{*}{ Dromiciops gliroides } & D. australis & 14 & 24 & $\mathrm{~A}$ & - & & Valdivia, Chile & Reig et al., 1972 \\
\hline & D. australis & 14 & 24 & A & A & & Valdivia, Chile & Reig et al., 1977 \\
\hline & D. australis & 14 & 24 & A & - & Ag-NOR & Valdivia, Chile & $\begin{array}{l}\text { Fernandez-Donoso et al., } \\
1979\end{array}$ \\
\hline & D. australis & 14 & 24 & A & UN & & $\begin{array}{l}\text { Valdivia, Osorno and } \\
\text { Concepción, Chile }\end{array}$ & $\begin{array}{l}\text { Gallardo and } \\
\text { Patterson, } 1987\end{array}$ \\
\hline & & 14 & 24 & ST & $\mathrm{D}$ & G & Valdivia and Osorno, Chile & Spotorno et al., 1997 \\
\hline
\end{tabular}

2n - diploid number; FN - fundamental number (number of autosomal arms); D - dot-like; A- acrocentric; T - telocentric; SM - submetacentric; M - metacentric; FISH - fluorescent in situ hybridization: Tel - telomere probe; rDNA - ribosomal DNA probe, Gen - whole genomic DNA probe; * - polymorphism of the X chromosome; UN - undistinguishable; a - current taxonomic names according to Wilson and Reeder, 2005; b - the provenience corresponds to states, departments or provinces in each country indicated; c - apud Hayman, 1990; $\mathrm{d}$ - in the original paper two Caenolestes species $(C$. obscurus and C. fulliginosus) were described with identical karyotypes. They were likely the same species as C. obscurus is now considered a synonym of C. fulliginosus.

ficult and variations in condensation complicates it even more.

The only cases of real intraspecific sex chromosome variation appear to be those of the $\mathrm{X}$ chromosomes of Gracilinanus emiliae, G. microtarsus, Marmosa murina and Thylamys elegans. Different morphologies were re- ported for these chromosomes by several authors (Table 1). Additionally, one specimen of Philander frenatus had a metacentric $\mathrm{Y}$, in contrast with the acrocentric form found otherwise in this species (Carvalho et al., 2002).

The Y chromosome was reported as undistinguishable or absent in males of Thylamys pallidior 
(Palma, 1995; Palma and Yates, 1996), Marmosops noctivagus, Thylamys elegans and Chironectes minimus (Palma and Yates, 1996). T. pallidior and M. noctivagus were only studied by these authors, but in C. minimus and T. elegans dot-like Y chromosomes were reported (Hayman and Martin, 1974; Reig et al., 1972, 1977). It is thus very likely that the four species above have dot-like Y chromosomes that were missed for technical reasons by Palma (1995) and Palma and Yates (1996). Another such case is that of Dromiciops gliroides, which was reported to have no $\mathrm{Y}$ chromosome in male bone marrow cells, that would have a $2 n=13$ (Gallardo and Patterson, 1987). Nevertheless, the $\mathrm{Y}$ chromosome of this species was also reported as dot-like in bone marrow cells in other studies (Reig et al., 1977; Spotorno et al., 1997) and was likely missed in the former report.

\section{Chromosome Banding In American Marsupials}

G-banding was performed in 14 species of American marsupials (Sinha and Kakati, 1976; Yonenaga-Yassuda et al., 1982; Merry et al., 1983; Rofe and Hayman, 1985; Casartelli et al., 1986; Souza et al., 1990; Pathak et al., 1993; Seluja et al., 1984; Spotorno et al., 1997; Svartman and Vianna-Morgante, 1999) (Table 1). No intraspecific variation was observed in these studies, which reinforced the conservation of the karyotypes extending it to G-banded chromosomes. Moreover, species with the same diploid number showed a striking similarity in their banded chromosomes with small karyotypic differences attributed to inversions and variation in the amount of constitutive heterochromatin (Yonenaga-Yassuda et al., 1982; Rofe and Hayman, 1985; Casartelli et al., 1986; Souza et al., 1990; Svartman and Vianna-Morgante, 1999). The comparison of G-banded karyotypes of species with $2 \mathrm{n}=14,18$ and 22 allowed to establish a complete homeology between all the autosomal arms of species with $2 \mathrm{n}=14,18$ and 22 (Svartman and Vianna-Morgante, 1998, 1999). This observation reinforced the role played by Robertsonian rearrangements in the karyotypical evolution of American marsupials (Svartman and Vianna-Morgante, 1998, 1999).

C-banding patterns were obtained in 22 species of American marsupials (references in Table 1) and, although intraspecific variation seemed absent, differences in constitutive heterochromatin contents and location were reported among species with the same diploid numbers. Most of the ten $2 n=14$ species studied presented pericentromeric heterochromatin in all autosomes and on the X chromosome (two species of Caluromys, two of Gracilinanus, Marmosa murina and Thylamys elegans). In Metachirus nudicaudatus only small pericentromeric C-bands were observed in the two smaller autosomes (pairs 5 and 6) and on the X chromosome. Large blocks of heterochromatin were present in the autosomes and on the $\mathrm{X}$ chromosomes of Marmosops incanus and M. parvidens. In M. incanus two additional large distal heterochromatic blocks were pres- ent, one in each arm of the metacentric $\mathrm{X}$ chromosome (Svartman and Vianna-Morgante, 1999; Carvalho et al., 2002; Pagnozzi et al., 2002). Micoureus demerarae had large heterochromatic blocks in the pericentromeric regions of the four largest autosome pairs (pairs 1-4) and very little pericentromeric $\mathrm{C}$-bands in the two smallest autosomes (pairs 5 and 6). Besides the pericentromeric C-band, the acrocentric $\mathrm{X}$ chromosome of this species also presented a distal heterochromatic block (Casartelli et al., 1986; Souza et al., 1990; Svartman and Vianna-Morgante, 1999; Pagnozzi et al., 2000; Carvalho et al., 2002). The Y chromosome was described as entirely heterochromatic in all the species.

Four of the five Monodelphis species studied ( $M$. brevicaudata, $M$. dimidiata, $M$. domestica and $M$. kunsi) presented pericentromeric heterochromatin in all autosomes and on the $\mathrm{X}$ chromosome and the tiny $\mathrm{Y}$ chromosome appeared completely heterochromatic. Monodelphis americana showed a different pattern, with pericentromeric heterochromatin restricted to the $\mathrm{X}$ chromosome and a completely heterochromatic Y (Merry et al., 1983, Svartman and Vianna-Morgante, 1999; Carvalho et al., 2002; Pagnozzi et al., 2002).

In all species with $2 \mathrm{n}=22$ studied the $\mathrm{Y}$ chromosome appeared heterochromatic. The most common pattern observed was the presence of C-bands in the pericentromeric regions of all autosomes and on the $\mathrm{X}$ chromosome, which was the case in Chironectes minimus, Didelphis albiventris, D. marsupialis and Philander frenatus. Pericentromeric heterochromatin was restricted to the $\mathrm{X}$ chromosome in Didelphis marsupialis, D. virginiana and Lutreolina crassicaudata. The two latter species presented distinctive features on their $\mathrm{X}$ chromosomes which had $\mathrm{C}$-banded blocks. This was also the case for the $\mathrm{X}$ chromosome of Philander opossum (Sinha et al., 1972; Sinha and Kakati, 1976; Yonenaga-Yassuda et al., 1982; Seluja et al., 1984; Casartelli et al., 1986; Svartman and Vianna-Morgante, 1999; Carvalho et al., 2002, Pagnozzi et al., 2002).

The variation in $\mathrm{X}$ chromosome size among species including the three diploid numbers was attributed to variations in the contents of constitutive heterochromatin (Yonenaga-Yassuda et al., 1982; Souza et al., 1990; Svartman and Vianna-Morgante, 1999).

Genome comparisons among Philander opossum $(2 \mathrm{n}=22)$, Micoureus demerarae and Marmosops incanus $(2 n=14)$ were extended through FISH (fluorescent in situ hybridization) using total genomic DNAs as probes (Svartman and Vianna-Morgante, 1999). In this study, interspecific conservation of the euchromatin among the three karyotypes was reinforced, while the heterochromatin was shown to be species-specific, not only in amount, but also in content.

Ag-NORs were studied in a total of 24 species of American marsupials. Thirteen of the species analyzed had a $2 \mathrm{n}=14$ (Caluromys lanatus, C. philander, Gracilinanus 
emiliae, G. microtarsus, Marmosa murina, Marmosops incanus, M. parvidens, M. paulensis, Metachirus nudicaudatus, Micoureus demerarae, M. paraguayensis, Thylamys elegans and Dromiciops australis; references in Table 1). All of these species presented Ag-NORs in the short arms of chromosome 6 (6p). In Dromiciops gliroides one additional NOR was observed in $5 p$ (Fernandez-Donoso et al., 1979) and in the two analyzed Micoureus species there were six NORs located on 5pq and 6p (Carvalho et al., 2002; Svartman and Vianna-Morgante, 2003).

Five species of Monodelphis, all with $2 \mathrm{n}=18$, had their Ag-NORs described ( $M$. brevicaudata, M. dimidiata, M. domestica, M. kunsi and M. rubida). Four of them presented a single NOR-bearing pair, which was $5 \mathrm{p}$ in three species (M. dimidiata, M. kunsi and M. rubida) and Xp in M. brevicaudata. Monodelphis domestica was the only species with $2 n=18$ to present four NORs, located on $5 p$ and $\mathrm{Xp}$ (references in Table 1). It is possible that all Monodelphis species have the same pattern observed in $M$. domestica (5p and $\mathrm{Xp}$ ) and that they were not detected because of the few specimens analyzed and because Ag-NORs reflect activity in the previous interphase and are thus variable. It would be interesting to perform FISH with an rDNA probe in order to determine the location of NORs in all the Monodelphis species. In Monodelphis domestica there is no inactivation of the NOR on the X chromosomes of the females, which are both active in every cell analyzed (Merry et al., 1983; Svartman and Vianna-Morgante, 2003). This may also be the case in M. brevicaudata (Carvalho et al., 2002).

Three species with $2 \mathrm{n}=22$ presented four Ag-NORs located on 5p7q (Lutreolina crassicaudata, Philander opossum and P. frenata), while Chironectes minimus had a single Ag-NOR on 5p. In Didelphis albiventris the maximum number of $\mathrm{Ag}$-NORs reported was six and they were located at the tip of the long arm of pairs 4, 5 and 6 (Seluja et al., 1984). Didelphis marsupialis had eight NORs as shown by FISH with an rDNA probe (Svartman and Vianna-Morgante, 2003), but the number of Ag-NORs reported was highly variable, possibly reflecting differences in activity between samples (Yonenaga-Yassuda et al., 1982; Casartelli et al., 1986; Carvalho et al., 2002; Svartman and Vianna-Morgante, 2003; Lima, 2004).

Besides Monodelphis domestica and D. marsupialis, other five species of American marsupials representing the three diploid numbers known in the group were studied after FISH with an rDNA probe (Svartman and ViannaMorgante, 2003). The results obtained, combined with the demonstration of G-banding homeologies between all the autosomal arms of the three diploid numbers $(2 \mathrm{n}=14,18$ and 22), led to the conclusion that at least one NOR-bearing pair is the same in the three different $2 \mathrm{n}$ complements. Thus, the NOR-bearing chromosome 6 of the $2 \mathrm{n}=14$ karyotype corresponds, respectively, to chromosomes 5 and 7 in the species with $2 \mathrm{n}=18$ and 22 , which also have NORs (Svartman and Vianna-Morgante, 2003).

\section{Karyotype Evolution In Marsupials}

The preponderance of a conserved karyotype with 14 chromosomes in animals of almost all marsupial families from the American and Australian faunas was interpreted as evidence that this was a conserved complement, possibly present in a common ancestor. Martin and Hayman (1967) and Hayman and Martin (1969) suggested that a karyotype with 14 chromosomes would be the "ancestral karyotype" present in an ancestral stock. It was suggested that the evolution proceeded from a karyotype with 14 chromosomes, which would have undergone centric fissions originating the second mode, a complement with 22 chromosomes, which in turn, would have given rise to the karyotypes with intermediate diploid numbers through centric fusions (Hayman, 1990). A karyotypical evolution through centric fissions from the basic karyotype with $2 \mathrm{n}=14$ was also suggested to explain the $2 \mathrm{n}=18$ and $2 \mathrm{n}=22$ in American marsupials (Reig et al., 1977).

An opposing view was held by Sharman (1973), who suggested that the second modal number of $2 n=22$ would be ancestral as it was present in the more plesiomorphic American didelphids and because chromosome fissions are rarer and more difficult to accept. This author thus proposed that chromosome fusions would explain the occurrence of marsupial karyotypes with diploid numbers lower than $2 \mathrm{n}=22$.

The later demonstration that the G-banding patterns in American and Australian species with $2 n=14$ were very similar, mainly differing due to intrachromosomal variations, was used to reinforce the idea that this was the marsupial "basic ancestral karyotype" and to lend support for the fission hypothesis (Rofe and Hayman, 1985; Hayman, 1990).

Recent studies using reciprocal interspecific chromosome painting to compare the chromosomes of marsupials from Australian and South American families confirmed the great conservation between chromosome segments among very divergent groups and allowed to delineate conserved segments that were differently combined in each species (Rens et al., 1999; 2001; 2003). These studies, which confirmed and extended the conclusions based on previous G-banding comparisons (Rofe and Hayman, 1985; Yonenaga-Yassuda et al., 1982; Souza et al., 1990; Svartman and Vianna-Morgante, 1999) have been frequently used in support of the fission hypothesis. Nevertheless, the demonstration of conservation does not in itself favor any of the two competing views.

The finding of interstitial telomeric sequences (ITS) in the autosomes of Didelphidae species with $2 \mathrm{n}=14$ and $2 \mathrm{n}=18$, but not in those with $2 \mathrm{n}=22$, led to the suggestion that the karyotype of South American marsupials would have proceeded through centric fusions leading to the re- 
duction in diploid number from an ancestral complement with at least 22 chromosomes (Svartman and Vianna-Morgante, 1998; Carvalho and Mattevi, 2000). This hypothesis defied the idea of the "ancestral" $2 \mathrm{n}=14$ marsupial karyotype which had prevailed for the previous three decades.

Pagnozzi et al. (2000) demonstrated that in Micoureus demerarae $(2 \mathrm{n}=14)$ autosomal pericentromeric interstitial telomeric sequences varied from 2 through 8 in specimens caught in different localities. These authors also stated that the ITS co-localized with heterochromatic blocks in $M$. demerarae and would thus represent part of satellite DNA sequences and not remnants of Robertsonian fusions. Pagnozzi et al. (2002) later performed the same kind of analysis in eight Didelphidae species, four with $2 \mathrm{n}=14$, two with $2 \mathrm{n}=18$ and two with $2 \mathrm{n}=22$, and came to a similar conclusion. These authors claimed that other kinds of datasets, basically derived from molecular studies involving DNA sequence comparisons, supported the ancestral $2 \mathrm{n}=14$ karyotype hypothesis and an evolution based on chromosome fissions would comply with it.

Taken together, the studies with ITS performed in American marsupials revealed conserved results in the six $2 \mathrm{n}=22$ species analyzed (Chironectes minimus, Didelphis albiventris, D. marsupialis, Philander frenatus, P. opossum and Lutreolina crassicaudata), that presented no ITS. In three out of the four species with $2 \mathrm{n}=18$ studied (Monodelphis brevicaudata, M. domestica and M. kunsi), ITS were conserved in the pericentromeric region of pair 1. The results in the eight $2 \mathrm{n}=14$ species analyzed were more variable: four species had no ITS (Caluromys philander, Metachirus nudicaudatus; Marmosa murina and Gracilinanus emiliae), Gracilinanus microtarsus had ITS on pair 1, Micoureus demerarae had a maximum of eight signals on the four largest autosome pairs, Marmosops incanus had ITS on pairs 1-5 and M. parvidens presented ITS in all of its six autosomal pairs (Svartman and ViannaMorgante, 1998; Carvalho and Mattevi, 2000; Pagnozzi et al., 2000, 2002).

It is interesting to point out that ITS were only observed in biarmed chromosomes. Another point to be stressed is that, with the exception of Marmosops incanus and Metachirus nudicaudatus studied by Svartman and Vianna-Morgante (1998), the results obtained by the other authors were from metaphases derived from bone marrow cells. These spreads present relatively condensed chromosomes when compared with those derived from cultured fibroblasts. A closer look at the results obtained by Svartman and Vianna-Morgante (1998) clearly show that the ITS detected by FISH in Marmosops incanus are close to but not inside the pericentromeric heterochromatin, as suggested by Pagnozzi et al. (2002).

Although the fusion hypothesis was clearly advanced to account for the karyotypic evolution of the three diploid numbers in American marsupials (Svartman and ViannaMorgante, 1998; Carvalho and Mattevi, 2000) Metcalfe et al. (2004) studied the distribution of ITS and of constitutive heterochromatin in nine species of Australian marsupials to test this view. Three of the species analyzed presented the presumed "ancestral" $2 n=14$ karyotype and the other six species were Macropodinae with varying diploid numbers which had been previously extensively studied. Centromeric ITS were observed in the largest three chromosome pairs of the $2 n=14$ species and also on pair 6 in two of them. In the Macropodinae species large ITS signals were observed in almost all chromosomes and the same sites were shown to be C-band positive. Macropus agilis, the only species without extensive C-banding, presented ITS in three pairs which corresponded to fusion sites from the putative $2 n=22$ ancestral macropodine complement. The authors associated the presence of most ITS with heterochromatin, but attributed the ITS observed in Macropus agilis to be the result from previously postulated chromosome fusions. Based on these results, the authors concluded that ITS represent telomeric sequences that are part of the native satellite DNA and that they only represent remnants of rearrangements when not present in the heterochromatin, a conclusion similar to that advanced by Pagnozzi et al. (2000, 2002).

In conclusion, it seems that the support given by various authors to each of the alternative hypotheses (fission or fusion) is strongly influenced by their view on how the chromosome data should be interpreted. Thus, those who believe that chromosome data are accessory to other kinds of datasets and should be used to corroborate them favor the fission hypothesis. Contrarily, authors that tend to interpret chromosome data independently before comparison with other kinds of studies are more inclined to support the fusion hypothesis. In any case, the resolution of this debate will rely on more detailed studies aiming at a molecular characterization of the ITS in marsupials. The sequencing of these regions could reveal if their composition is strictly telomeric or if they are part of satellite DNAs located at the heterochromatin. Another possibility is to verify if the composition of the satellite DNAs present in the heterochromatin of different species is similar and contain telomere sequences embedded in them.

\section{Marsupials and Sex Chromosomes}

Among the main contributions resulting from marsupial genetics studies are those related to sex chromosomes evolution and function. Sex determination in eutherians depends on the presence of the $\mathrm{Y}$ chromosome. Testis development is primarily due to the $S R Y$ gene and other male characters develop later under the control of the testicular hormones. Testes development in marsupials is also determined by the $\mathrm{Y}$ chromosome, which bears an orthologue of the human SRY gene (Foster et al., 1992). Nevertheless, as demonstrated by Shaw et al. (1990), in marsupials the scrotum develops before the testes and thus, differently from 
eutherians, scrotum differentiation is not dependent on the hormones secreted by the developed testis.

Like most mammals, the majority of marsupials present a chromosome sex determination system of the XX:XY type. Multiple sex chromosomes systems were only described in four Australian species: in Potorous tridactylus, Wallabia bicolor and Macrotis lagotis the multiple sex chromosomes result from a translocation of an autosome to the X chromosome, whereas Lagorchestes conspicillatus presents a more complex system, involving translocations between two autosomes and both sex chromosomes (reviewed in Hayman, 1990).

Sex chromosome mosaicism resulting from their elimination in somatic tissues was described in several species. Almost all specimens from the Australian family Peramelidae (genus Echymipera, Isoodon, Perameles and Peroryctes) analyzed had one $\mathrm{X}$ chromosome eliminated in females and the $\mathrm{Y}$ chromosome eliminated in the same somatic tissues in males. The $\mathrm{Y}$ chromosome was absent in most cells of the bone marrow, liver and spleen of Petauroides volans (family Petauridae), but cells of the same tissue retained both $\mathrm{X}$ chromosomes in females (Murray and McKay, 1979).

A mechanism similar to that of $P$. volans, with the absence of the $\mathrm{Y}$ chromosome in bone marrow cells, was described in the American marsupial Dromiciops gliroides and used as evidence of its close phylogenetic relationship to Australian marsupials (Gallardo and Patterson, 1987). Nevertheless, the tiny Y chromosome of $D$. gliroides was probably missed by the authors, as discussed above in a previous topic.

Some cytological features of marsupial sex chromosomes are peculiar: the very small $\mathrm{Y}$ chromosome, which is dot-like in many species, and the small size of the $\mathrm{X}$ chromosome (3\% of the genome) in relation to the eutherian $\mathrm{X}$ (5\% of the genome) (Hayman et al., 1982); the absence of the synaptonemal complex between the $\mathrm{X}$ and $\mathrm{Y}$ chromosomes during meiosis, which may be due to the absence of the pseudoautosomal region in marsupials (Sharp, 1982); the presence of nucleolus organizer regions (NORs) in the $\mathrm{X}$ or in both sex chromosomes of some species without their inactivation on one of the $\mathrm{X}$ chromosomes in females, which implies in escape from inactivation and no dosage compensation for these loci (Hayman, 1990; Svartman and Vianna-Morgante, 2003); and the differential location of chiasmata in the meiosis of males and females of some species (interstitial chiasmata in males and distal chiasmata in females, resulting in a much higher recombination rate in males), as Sminthopsis crassicaudata and Monodelphis domestica (Bennett et al., 1986; Hayman et al., 1988; Samollow et al., 2004).

The absence of a synaptonemal complex between the $\mathrm{X}$ and $\mathrm{Y}$ chromosomes during the first meiotic prophase in males of the South American marsupials Thylamys elegans, Dromiciops gliroides and Rhyncholestes raphanarus, was demonstrated by Page et al. (2003, 2005). Instead, a structure called dense plate, a modification of the axial elements of the sex chromosomes, forms between the $\mathrm{X}$ and $\mathrm{Y}$ chromosomes during pachytene, thus ensuring their correct segregation.

The inactivation of the $\mathrm{X}$ chromosome in marsupials also presents important differences in relation to that of eutherians. In the somatic cells of eutherian females one X chromosome is randomly inactivated. This inactivation is stable through cell generations and encompasses most genes on the $\mathrm{X}$ chromosome. In marsupials and monotremes, there is preferential inactivation of the paternal $\mathrm{X}$ chromosome, which is incomplete and tissue specific (Cooper et al., 1990; Heard and Disteche, 2006). The mechanisms involved in this inactivation are just starting to be understood (Hornecker et al., 2007; Namekawa et al., 2007).

Among the studies performed in marsupials, gene mapping was especially fruitful in providing important information related to chromosome evolution in mammals, particularly about the sex chromosomes.

Marsupials were very important in the identification of the mammalian testis determining factor (TDF) on the $\mathrm{Y}$ chromosome. The autosomal location of the putative TDF, the $Z F Y$ gene, in marsupials, was a strong indication that this was not the TDF gene (Sinclair et al., 1988). The later finding of $S R Y$, another candidate for TDF, on the marsupial Y chromosome, was used as evidence to corroborate its role as the mammalian TDF (Foster et al., 1992).

Mapping of human X-linked genes in marsupials resulted in important findings. Genes already mapped in the human Xq were also mapped in the marsupial and monotreme $\mathrm{X}$ chromosomes, defining a mammalian $\mathrm{X}$-conserved region (XCR). Nevertheless, human Xp genes were located in three autosomal regions in both marsupials and monotremes (reviewed in Wilcox et al., 1996 and in Graves, 2006).

Based on the sex chromosomes mapping data and behavior in the three mammalian groups, Graves (1995) suggested that the eutherian sex chromosomes evolved through cycles of autosome additions to the ancestral $\mathrm{X}$ and $\mathrm{Y}$ chromosomes followed by the degradation of Y chromosome sequences and the recruitment of the equivalent regions on the $\mathrm{X}$ by the inactivation mechanism. The pseudoautosomal region on the human sex chromosomes would be a relict of the last addition and the genes on the $\mathrm{Y}$ chromosome without an essential function in sex determination and differentiation in males would tend to mutate or to be deleted (Graves, 1995; reviewed in Graves, 2006).

Gene mapping results in marsupials contradicted the hypothesis of mammalian $\mathrm{X}$ chromosome conservation proposed by Ohno (1967), as only part of the X was conserved in mammals. The same results also weakened the idea proposed by Lyon (1974) that the loss of Y chromosome genes and $\mathrm{X}$ chromosome inactivation could have 
happened in a single deletion or translocation event, as suggested by the lack of variation in size and gene content of the mammalian $\mathrm{X}$ chromosome.

Gene mapping data in American marsupials were much more limited than those from Australian species. Until a decade ago, Didelphis virginiana was the only American marsupial species to have genes mapped by in situ hybridization. In this case, G6PD was located on the Xp and HPRT was mapped on the Xq (Driscoll and Migeon, 1988). These genes are in the region considered to be conserved on the $\mathrm{X}$ chromosome of all species of eutherians, marsupials and monotremes already studied. They are closely located in eutherians and more distantly located in marsupials. In most species of Dasyuridae and Didelphidae the $\mathrm{X}$ chromosome is a small acrocentric, considered to be the marsupial ancestral X (Rofe and Hayman, 1985). The X chromosome of $D$. virginiana is submetacentric and probably originated from a pericentric inversion in the $D$. marsupialis $\mathrm{X}$ chromosome, which would explain the location of G6PD and HPRT in different $\mathrm{X}$ chromosome arms (Driscoll and Migeon, 1988).

Recently, the lack of more detailed data from American marsupials changed radically with the publication of the genome sequence of Monodelphis domestica, the first marsupial species to be sequenced (Mikkelsen et al., 2007). This species has been used as a model laboratory animal for biomedical research for some decades and, although originally from South America, has been studied by scientists worldwide. The contributions provided by the study of $M$. domestica for different biological areas along the years were recently reviewed by Samollow (2008) and include very important data on mammalian genetics.

In conclusion, studies already performed in marsupials, particularly in Australian species, have demonstrated the great potential provided by the group for the understanding of basic genetic mechanisms in mammals. Genetic studies in American marsupials are relatively scarce and cytogenetic data of most species are restricted to karyotype description, usually without banding patterns. We hope to see this situation change by having South American researchers interested in studying marsupials, which are not only an important component of our biodiversity, but a treasure trove of information on genome function and evolution.

\section{References}

Beck RMD, Godthelp H, Weisbecker V, Archer M and Hand SJ (2008) Australia's oldest marsupial fossils and their biogeographical implications. PLoS ONE 3:e1858.

Bennett JH, Hayman DL and Hope RM (1986) Novel sex difference in linkage values and mitotic chromosome behaviour in a marsupial. Nature 233:59-60.

Biggers JD, Fritz HI, Hare WC and McFeely RA (1965) Chromosomes of American marsupials. Science 148:1602-1603.

Bininda-Emonds ORP, Cardillo M, Jones KE, MacPhee RDE, Beck RMD, Grenyer R, Price SA, Vos RA, Gittleman JL and Purvis A (2007) The delayed rise of present-day mammals. Nature 446:507-512.

Carvalho BD and Mattevi MS (2000) $\left(\mathrm{T}_{2} \mathrm{AG}_{3}\right)$ n telomeric sequence hybridization suggestive of centric fusion in karyotype marsupials evolution. Genetica 108:205-210.

Carvalho BA, Oliveira LFB, Nunes AP and Mattevi MS (2002) Karyotypes of nineteen marsupial species from Brazil. J Mammal 83:58-70.

Casartelli C, Rogatto SR and Ferrari I (1986) Cytogenetic analysis of some Brazilian marsupials (Didelphidae, Marsupialia). Can J Genet Cytol 28:21-29.

Clemens WA (1977) Phylogeny of marsupials. In: Stonehouse B and Gilmore D (eds) The Biology of Marsupials. University Park Press, Baltimore, pp 51-68.

Cooper DW, Johnston PG, VandeBerg JL and Robinson ES (1990) X-chromosome inactivation in marsupials. Aust J Zool 37:411-417.

Dawson JD (1983) Marsupials: Origins and historical biogeography. In: Edward Arnold (ed) Monotremes and Marsupials: The Other Mammals. Edward Arnold, London, pp 33-38.

Driscoll DJ and Migeon BR (1988) Localization of G6PD and HPRT to different arms of the X chromosome of the North American marsupial (Didelphis virginiana) by in situ hybridization and deletion mapping: Evolutionary significance. Genomics 3:308-314.

Engstrom MD and Gardner AL (1988) Karyotype of Marmosa canescens (Marsupialia, Didelphidae): A mouse opossum with 22 chromosomes. South Nat 33:231-233.

Fernandez-Donoso R, Berrios S and Pinchera J (1979) Position of the nucleolus within the nuclei of spermatocytes of Dromiciops australis and Marmosa elegans (Didelphoidea-Marsupialia). Experientia 35:1021-1023.

Foster JW, Brennan FE, Hampikian GK, Goodfellow PN, Sinclair AH, Lovell-Badge R, Cooper DW and Graves JAM (1992) Evolution of sex determination and the Y chromosome: SRY-related sequences in marsupials. Nature 359:531-533.

Gallardo MH and Patterson BD (1987) An additional 14-chromosome karyotype and sex-chromosome mosaicism in South American marsupials. Fieldiana Zool 1382:111-115.

Graves JAM (1995) The origin and function of the mammalian Y chromosome and Y- borne genes - An evolving understanding. BioEssays 17:311-321.

Graves JAM (2006) Sex chromosome specialization and degeneration in mammals. Cell 124:901-914.

Hayman DL (1990) Marsupial cytogenetics. Aust J Zool 37:331349.

Hayman DL and Martin PG (1969) Cytogenetics of marsupials. In: Benirschke K (ed) Comparative Mammalian Cytogenetics. Springer-Verlag, New York, pp 191-217.

Hayman DL and Martin PG (1974) Mammalia I: Monotremata and Marsupialia, Chordata 4. In: John B (ed) Animal Cytogenetics, Gebrüder Borntraeger, Berlin, $110 \mathrm{pp}$.

Hayman DL, Ashworth LK and Carrano AV (1982) The relative DNA content of the eutherian and marsupial X chromosome. Cytogenet Cell Genet 34:265-270.

Hayman DL, Kirsch JA, Martin PG and Waller PF (1971) Chromosomal and serological studies of the Caenolestidae and their implications for marsupial evolution. Nature 231:194195. 
Hayman DL, Moore HDM and Evans EP (1988) Further evidence of novel sex differences in chiasma distribution in marsupials. Heredity 61:455-458.

Heard E and Disteche CM (2006) Dosage compensation in mammals: Fine-tuning the expression of the $\mathrm{X}$ chromosome. Genes Dev 20:1848-1867.

Hornecker JL, Samollow PB, Robinson ES, VandeBerg JL and McCarrey JR (2007) Meiotic sex chromosome inactivation in the marsupial Monodelphis domestica. Genesis 45:696708

Langguth A and Lima FS (1988) The karyotype of Monodelphis americana (Marsupialia - Didelphidae). Revta Nordest Biol 6:1-5.

Lima FS (2004) Cariótipos e regiões organizadoras de nucléolos (RON) de Marmosa e Didelphis (Didelphidae) do estado do Tocantins, Brasil. The karyotype of Monodelphis americana (Marsupialia - Didelphidae). Revta Nordest Biol 18:87-93.

Luo ZX, Ji Q, Wible JR and Yuan CX (2003) An early Cretaceous tribosphenic mammal and metatherian evolution. Science 302:1934-1940.

Lyon MF (1974) Mechanisms and evolutionary origins of variable X-chromosome activity in mammals. Proc R Soc London B 187:243-268.

Martin PG and Hayman DL (1967) Quantitative comparisons between the karyotypes of Australian marsupials from three different superfamilies. Chromosoma 20:290-310.

Merry DE, Pathak S and VandeBerg JL (1983) Differential NOR activities in somatic and germ cells of Monodelphis domestica (Marsupialia, Mammalia). Cytogenet Cell Genet 35:244-251.

Metcalfe CJ, Eldridge MD and Johnston PG (2004) Mapping the distribution of the telomeric sequence $\left(\mathrm{T}_{2} \mathrm{AG}_{3}\right)_{\mathrm{n}}$ in the $2 \mathrm{n}=$ 14 ancestral marsupial complement and in the macropodines (Marsupialia, Macropodidae) by fluorescence in situ hybridization. Chromosome Res 12:405-414.

Mikkelsen TS, Wakefield MJ, Aken B, Amemiya CT, Chang JL, Duke S, Garber M, Gentles AJ, Goodstadt L and Heger A (2007) Genome of the marsupial Monodelphis domestica reveals innovation in non-coding sequences. Nature 447:167178.

Murray JD and McKay GM (1979) Y chromosome mosaicism in pouch young of the marsupial, greater glider (Marsupialia, Petauridae). Chromosoma 72:329-334.

Namekawa SH, VandeBerg JL, McCarrey JR and Lee JT (2007) Sex chromosome silencing in the marsupial male germ line. Proc Natl Acad Sci USA 104:9730-9735.

Nowak RM (1991) Order Marsupialia. In: Nowak RM (ed) Walker's Mammals of the World. 5th edition. The Johns Hopkins University Press, Baltimore, pp 10-113.

Ohno S (1967) Sex Chromosomes and Sex Linked Genes. Springer Verlag, Berlin, 192 pp.

Page J, Berríos S, Parra MT, Viera A, Suja JA, Prieto I, Barbero JL, Rufas JS and Fernández-Donoso R (2005) The program of sex chromosome pairing in meiosis is highly conserved across marsupial species: Implications for sex chromosome evolution. Genetics 170:793-799.

Page J, Berríos S, Rufas JS, Parra MT, Suja JA, Heyting C and Fernández-Donoso R (2003) The pairing of X and Y chromosomes during meiotic prophase in the marsupial species Thylamys elegans is maintained by a dense plate developed from their axial elements. J Cell Sci 116:551-560.
Pagnozzi JM, Ditchfield AD and Yonenaga-Yassuda Y (2002) Mapping the distribution of the interstitial telomeric (TTAGGG)n sequences in eight species of Brazilian marsupials (Didelphidae) by FISH and the correlation with constitutive heterochromatin. Do ITS represent evidence for fusion events in American marsupials? Cytogenet Genome Res 98 278-284.

Pagnozzi JM, Silva MJJ and Yonenaga-Yassuda Y (2000) Intraspecific variation in the distribution of the interstitial telomeric (TTAGGG)n sequences in Micoureus demerarae (Marsupialia, Didelphidae) Chromosome Res 8:585-591.

Palma RE (1995) The karyotypes of two South American mouse opossums of the genus Thylamys (Marsupialia, Didelphidae), from the Andes, and eastern Paraguay. Proc Biol Soc Washington 108:1-5.

Palma RE and Yates TL (1996) The chromosomes of Bolivian didelphid marsupials. Occas Papers Mus Texas Tech Univ 162:1-20.

Paresque R, Souza WP, Mendes SL and Fagundes V (2004) Composição cariotípica da fauna de roedores e marsupiais de duas áreas de Mata Atlântica do Espírito Santo, Brasil. Bol Mus Biol Mello Leitão 17:5-33.

Pathak S, Rønne M, Brown NM, Furlong CL and VandeBerg JL (1993) A high-resolution banding pattern idiogram of Monodelphis domestica chromosomes (Marsupialia, Mammalia). Cytogenet Cell Genet 63:181-184.

Pereira LG and Geise L (2007) Karyotype composition of some rodents and marsupials from Chapada Diamantina (Bahia, Brasil). Braz J Biol 67:509-518.

Pereira NP, Ventura K, Silva Júnior MC, Silva DM, YonenagaYassuda Y and Pellegrino KCM (2008) Karyotype characterization and nucleolar organizer regions of marsupial species (Didelphidae) from areas of Cerrado and Atlantic Forest in Brazil. Genet Mol Biol 31:887-892.

Reig OA (1968) The chromosomes of the didelphid marsupial Marmosa robinsonii Bangs. Experientia 24:185-186.

Reig OA and Bianchi NO (1969) The occurrence of an intermediate didelphid karyotype in the short-tailed opossum (genus Monodelphis). Experientia 25:1210-1211.

Reig OA and Sonneschein C (1970) The chromosomes of Marmosa fuscata Thomas, from northern Venezuela (Marsupialia, Didelphidae). Experientia 26:199-200.

Reig OA, Fernández-Donoso R and Spotorno OA (1972) Further occurrence of a karyotype of $2 n=14$ chromosomes in two species of Chilean didelphoid marsupials. Z Saugetierkd $37: 37-42$.

Reig OA, Gardner AL, Bianchi NO and Patton JL (1977) The chromosomes of the Didelphidae (Marsupialia) and their evolutionary significance. Biol J Linn Soc 9:191-216.

Rens W, O'Brien PC, Fairclough H, Harman L, Graves JA and Ferguson-Smith MA (2003) Reversal and convergence in marsupial chromosome evolution. Cytogenet Genome Res 102:282-290.

Rens W, O'Brien PCM, Yang F, Graves JAM and FergusonSmith MA (1999) Karyotype relationships between four distantly related marsupials revealed by reciprocal chromosome painting. Chromosome Res 7:461-474.

Rens W, O’Brien PC, Yang F, Solanky N, Perelman P, Graphodatsky AS, Ferguson MW, Svartman M, De Leo AA, Graves JA, et al. (2001) Karyotype relationships between 
distantly related marsupials from South America and Australia. Chromosome Res 9:301-308.

Rofe R and Hayman D (1985) G-banding evidence for a conserved complement in the Marsupialia. Cytogenet Cell Genet 39:40-50.

Samollow PB (2008) The opossum genome: Insights and opportunities from an alternative mammal. Genome Res 18:11991215.

Samollow PB, Kammerer CM, Mahaney SM, Schneider JL, Westenberger SJ, VandeBerg JL and Robinson ES (2004) First generation linkage map of the gray, short-tailed opossum, Monodelphis domestica, reveals genome-wide reduction in female recombination rates. Genetics 166:307-329.

Seluja GA, Di Tomaso MV, Brum-Zorrilla N and Cardoso H (1984) Low karyotypic variation in two didelphids (Marsupialia): Karyogram and chromosome banding analysis. J Mammal 65:702-707.

Sharman GB (1973) The chromosomes of non-eutherian mammals. In: Chiarelli AN and Capanna E (eds) Cytotaxonomy and Vertebrate Evolution. Academic Press, New York, pp 485-530.

Sharp P (1982) Sex chromosome pairing during male meiosis in marsupials. Chromosoma 86:27-47.

Shaw G, Renfree MB and Short RV (1990) Primary genetic control of sexual differentiation in marsupials. Aust J Zool 37:443-450.

Sinclair AH, Foster JW, Spencer JA, Page DC, Palmer M, Goodfellow PN and Graves JAM (1988) Sequences homologous to ZFY, a candidate human sex-determining gene, are autosomal in marsupials. Nature 336:780-783.

Sinha AK and Kakati S (1976) C- and G-bands of the opossum chromosomes: Terminal sequences of DNA replication. Can J Genet Cytol 18:195-205.

Sinha AK, Kakati S and Pathak S (1972) Exclusive localization of C-bands within opossum sex chromosomes. Exp Cell Res 75:265-267.
Souza MJ, Maia V and Santos JF (1990) Nucleolar organizer regions, G- and C-bands in some Brazilian species of Didelphidae. Braz J Genet 13:767-775.

Spotorno AE, Marin JC, Yévenes M, Walker LI, FernándezDonoso R, Pincheira J, Berríos MS and Palma RE (1997) Chromosome divergence among American marsupials and the Australian affinities of the American Dromiciops. J Mammal Evol 4:259-269.

Stonehouse WA (1977) Introduction: The Marsupials. In: Stonehouse B and Gilmore D (eds) The Biology of Marsupials. University Park Press, Baltimore, pp 1-5.

Svartman M and Vianna-Morgante AM (1998) Karyotype evolution of marsupials: From higher to lower diploid numbers. Cytogenet Cell Genet 82:263-266.

Svartman M and Vianna-Morgante AM (1999) Comparative genome analysis in American marsupials: Chromosome banding and in-situ hybridization. Chromosome Res 7:267-275.

Svartman M and Vianna-Morgante AM (2003) Conservation of chromosomal location of nucleolus organizer in American marsupials (Didelphidae). Genetica 118:11-16.

Wilcox SA, Watson JM, Spencer JA and Graves JAM (1996) Comparative mapping identifies the fusion point of an ancient mammalian X-autosomal rearrangement. Genomics 35:66-70.

Wilson DE and Reeder DM (2005) Mammal Species of the World: A Taxonomic and Geographic Reference. 3rd edition. Johns Hopkins University Press, Baltimore, 2142 pp.

Yonenaga-Yassuda Y, Kasahara S, Souza MJ and L'Abbate M (1982) Constitutive heterochromatin, G-bands and nucleolus-organizer regions in four species of Didelphidae (Marsupialia). Genetica 58:71-77.

Yunis E, Cayon J and Ramirez E (1973) The chromosomes of Metachirus nudicaudatus (Marsupialia, Didelphidae). Aust J Zool 21:369-373.

Associate Editor: Yatiyo Yonenaga-Yassuda

License information: This is an open-access article distributed under the terms of the Creative Commons Attribution License, which permits unrestricted use, distribution, and reproduction in any medium, provided the original work is properly cited. 